\title{
Reflecting on what 'you said' as a way of reintroducing difficult topics in child mental health assessments
}

\begin{abstract}
Background: In child and adolescent mental health assessments questions are integral to the process. There has been limited research focused on the assessment process, or on how questions are constructed within this clinical environment.

Methods: We examined 28 naturally-occurring initial assessments, with particular attention to how practitioners used questions in their communication with children and young people. We utilised conversation analysis to examine the data.

Results: Analysis revealed a particular type of question preface used to reintroduce a prior topic. This was achieved through the use of 'you said x' as a foundation for asking a followup question and demonstrated active listening.

Conclusions: Arguably, this approach is a useful way of gathering assessment-relevant information in a child-centred way.
\end{abstract}

Key words: Communication, mental health, conversation analysis, children, questions, assessment, active listening

Key practitioner message:

- It is well-known that outcomes of clinical practice are better when children are appropriately engaged in the process. 
- However, children may find it challenging to discuss difficult topics in mental health settings.

- Our findings have shown that when something the child has talked about previously is reintroduced, it provides a legitimate way to ask for further elaboration.

- In particular, the data revealed a three-part sequence which was effective in eliciting information from children.

- These findings have relevance for clinical practice, as practitioners can easily incorporate this technique into their own work in child and adolescent mental health. 


\title{
Reflecting on what 'you said' as a way of reintroducing difficult
}

\author{
topics in child mental health assessments
}

\section{Introduction}

A central feature of clinical practice is the information-gathering process which is central to good communication. In child mental health in particular, the skilful questioning of children is essential in informing clinical decisions. Research has demonstrated that practitioners may have difficulty eliciting relevant answers from children (Stivers, 2001). However, guiding policies advocate child-centred practice, which emphasises the value of listening to the child's voice. Evidence indicates that greater engagement and involvement of children, leads to better results (Chu \& Kendall, 2004). In practice there are several verbal ways of demonstrating understanding of what the client has said, such as summarising and reflecting. These are important skills that can be utilised to check understanding and display active listening. In child mental health settings, active listening refers to the way that practitioners indicate responsivity to what the child has said (Hutchby, 2005). Active listening in clinical communication therefore is one way that practitioners choose their words carefully for therapeutic purposes. Often central to this communication with children is the use of questions.

The considered design of questions can serve the purposes of building engagement through conveying attentiveness to the child's words. Despite questions being intrinsic to communication in clinical practice there is little empirical literature exploring this area (Ziolkowska, 2009). However, contemporary evidence suggests that practitioners employ a 
wide range of questioning styles (O’Reilly, Karim, \& Kiyimba., 2015). The research conducted has predominantly come from communication studies, and in particular studies utilising conversation analysis (CA), which attends to the specific language used. CA benefits from utilising naturally-occurring data and is able to illuminate actual practice (O’Reilly, Karim, Stafford et al., 2015). This type of research highlights how powerful language can be and how influential the choices of specific words can be to the trajectory of the conversation. One area where this of relevance is question design.

Question design is especially important for the initial consultation as questioning is the primary way of gathering sufficient information to achieve the goals of the assessment. Typically, assessments involve sequences of questions and answers as practitioners seek to clarify mental health need (see for example, Antaki \& O’Reilly, 2014; McCabe, Heath, Burns et al, 2002). However, there has been limited research evidence examining assessments (Mash \& Hunsley, 2005), particularly using qualitative approaches that focus on communication (Hartzell, Seikkula, \& von Knorring., 2010).

The purpose of assessments is to screen for mental health difficulties in children who are usually referred to Child and Adolescent Mental Health Service (CAMHS) (Parkin, Frake \& Davidson., 2003). There are multiple functions to the assessment including, building relationships, gathering information, and determining service requirements. However, this information-gathering task must be contextualised within the framework that this is the first time the practitioner has met the child and their family, and that engaging children in discussing potentially difficult or sensitive topics can be challenging. Furthermore, research has demonstrated that children tend to feel quite anxious prior to attending CAMHS appointments (Bone et al., 2015). The crafting of questions in a way that engages rather than 
alienates children in this artificial and probably stressful situation is therefore paramount to the interaction. Evidence that demonstrates the skilful crafting of questions in a way that validates and engages children, while still managing to elicit assessment-relevant information is likely to be of interest to practitioners.

\section{Aims of the paper}

The aims of this research were to explore conversational mechanisms for engaging children in talking about difficult topics. We examined how practitioners reflected the words or phrases that a child had introduced earlier in the session as a way to reintroduce that topic into the current conversation. Specifically, the focus for the paper is on how the question preface ‘you said’ was used.

Context and Setting

The data consisted of video-recorded child mental health assessments with 28 families attending a UK CAMHS. Each appointment lasted approximately ninety minutes. All families, except one, were seen by two practitioners who were child and adolescent psychiatrists (trainee and consultant), clinical psychologists, assistant psychologists, community psychiatric nurses (CPN's), learning disabilities nurses, occupational therapists (OTs) and psychotherapists. Some sessions included medical students (1) and/or student nurses (2). 
Data were representative of attendance at CAMHS and included 64\% boys and 36\% girls, aged 6-17 years (Mean 11.21, SD = 3.10). Twenty-seven children attended with their mothers, seven also with fathers (one child attended only with their father). Six of the children’s maternal grandmothers were present.

\section{Data analysis}

The assessments were analysed using conversation analysis (CA) which pays close attention to the interactional details. CA is a well-established and robust methodological approach to the study of talk-in-interaction (see Heritage, 1984). CA favours the use of naturallyoccurring data, which is capturing events that occur naturally regardless of research involvement (Hutchby \& Woffitt, 2008). This has the benefit of showing what actually happens in practice rather than retrospective reports typically gathered through interviewing or focus groups (Potter, 2002).

As an observational science, CA is based on directly observable characteristics of the data rather than subjective interpretations (Drew, Chatwin \& Collins, 2001). It is an increasingly popular methodology used for studying interactions between doctors and patients (Stivers, 2002) and mental health interactions (O’Reilly \& Lester, 2015). The process of analysis involves gathering occurrences of similar interactional practices within the data corpus to determine recurrent or systematic communication patterns (Drew et al., 2001). In practice this means that the analyst brings together a corpus of extracts with similar features and identifies the sequential patterns in the talk. This process is data-driven where analytic claims are evidenced through the data and is facilitated by researcher co-analysis to promote 
methodological rigour and objectivity. CA utilises a detailed transcription system that represents intonation, pauses and volume (Jefferson, 2004).

\section{Ethics}

Ethical approval was granted by the UK National Research Ethics Service. Informed consent was acquired by all parties prior to and immediately after the assessment and all participants were able to withdraw from the study.

\section{Results}

Child mental health assessments are characterised by asking questions in order to ascertain diagnostic or service relevant information. However, in unfamiliar environments children may find it difficult to answer personal questions (Parker \& O’Reilly, 2012). We observed that practitioners used the phrase 'you said' as a way to introduce topics that had been mentioned earlier by the child. Usually this phrase was followed by either a paraphrase or reiteration of the exact words used. The use of this ‘you said’ preface before a question was shown to be effective in eliciting further information from the child about the re-introduced topic. The term 'effective' in this context relates to; a) a response being given; b) the response being relevant to the question asked and; c) the response containing detail. Of the 108 instances where 'you said' was used as a preface to a question, all instances of its use were effective in eliciting appropriate detail. Using an inductive ('bottom-up') approach, we investigated the sequences of talk which included a 'you said' preface, question and answer. There has been little research on the properties of 'you said' formulations (Wooffitt \& 
Allistone, 2008), although there have been several studies examining the qualities and functions of what has been described as 'reported speech'.

Reported speech has a number of features which distinguish it, namely that it is displayed as sounding the same (through intonation, inflection and pace) as the original saying and typically utilises third person references such as 'he said-she said' (Holt, 1996). For example, a clinician may attend a team meeting after talking to a colleague the previous day who is unable to attend it. They may 'report' what that colleague said in the format of he said "I have another meeting that finishes at 11 across town, but will try to drop in”. In this example of reported speech, the words of the colleague are presented as if they are the exact phrase spoken. The research on reported speech is based on 'here-and-now' conversations, where the elements of speech reported are separated from the original environment. In other words, the speech originally uttered occurred at a different time and place to the one in which it is being reported, and were spoken by another person, other than those in the current interaction.

We make a distinction between 'reported speech' which is typically prefaced by he-said or she-said and 'reflected speech', which is typically prefaced by 'you said'. Reflected speech is distinguished as being used in the presence of the individual whose words are being reported, without any attempt to sound (in pitch or style) like the original version. In the data we found that there were consistent features in the way that reflected speech was introduced into the conversation using a 'you said' preface. We also investigated the function of questions using this 'you said' preface and found that the 'you said' was used to reintroduce a previous topic and to provide a platform for asking follow-up questions based on that topic. 


\section{The reflected speech sequence}

A recurrent sequential pattern was identified. This sequence consisted of three parts; 1) 'you said’ preface and reflected speech; 2) recipient response slot (opportunity for recipient to respond); 3) follow-up question-answer adjacency pair (this is summarised in table 1). An adjacency pair is a well-established component of talk recognised within CA. This refers to the common occurrence of certain components of talk occurring together (Sacks, 1992). For example, a question is usually followed by an answer and thus forms a type of adjacency pair.

Table 1: The three components of the sequence

1. 'You said' preface plus reflected speech.

2. Recipient response slot.

3. Question-answer adjacency pair.

Extract one: Family ten $($ Prac $=$ Clinical Psychologist $)$

Prac

you used an imıportant word earlier you said er they provoke $\downarrow$ me

$(0.78)$

Prac

um $\mathrm{W} W$ : what $\downarrow$ happens how would they pro $\downarrow$ voke you?

Child they: start to $\downarrow$ call me names

This extract illustrates the three-part sequence:

1. You said preface plus reflected speech: "you said er they provoke me"

2. Recipient response slot: Pause of (0.78) seconds to allow child to respond. 
3. Question: “what happens, how would they provoke you?” Followed by the answer:

"They start to call me names"

Following part one of the sequence, 'you said er they provoke me' there was a significant pause '(0.78)'. In CA, where there are naturally occurring opportunities within the conversation for another person to speak, these are known as transition relevance places (TRP) (Sacks, Schegloff \& Jefferson, 1974). One of the ways that these are recognisable is by the 'completing' intonation used by the person speaking just before the TRP. In the sequence that we have presented, downward intonation (marked by a downward arrow - $\downarrow$ me) is one way of representing completing intonation. At this point we have referred to this recognisable opportunity for the child to respond as a recipient response slot. In some instances, the child responded, and at other times (such as this instance) they did not. Typically, in conversation where the recipient remains silent at a TRP, this is treated by the speaker as tacit agreement. In this extract, in the absence of a verbal response from the child, the practitioner continued the sequence by introducing a question, which implicitly treated the silence as agreement.

Extract two: Family ten $($ Prac $=$ Clinical psychologist $)$

Prac

and uh: you said the cause of $\downarrow$ that is that you get bored

$(0.73)$

Prac
an: $d$ um $(\odot .68) i$-is there $\downarrow$ anything else you could
$\downarrow$ do to to: kind of $(0.53)$ beat the $\downarrow$ boredom $(0.67)$
an- any activities $\downarrow$ or (0.39)er tell your parents
talk to them and $\downarrow$ say you know I'm bored an' ( $\odot .33)$
want something to $\downarrow$ do: $(\odot .96) \downarrow$ or $I^{\prime} m$ lonely: 

$\uparrow$ work

The three-part sequence is again evident in this extract.

1. You said preface plus reflected speech: "you said the cause of that is that you get bored"

2. Recipient response slot: A pause of (0.73) seconds to allow the child to respond.

3. Question: “is there anything else you could do to to kind of beat the boredom?"

Followed by the answer: "If I get bored my mum's going to tell me to do some work" In this example the question element of the sequence was again constructed in a traditional question format 'is there anything'. In addition, although the question was extended because it contained some suggested answers, the child provided the normative second part of the question-answer adjacency pair by providing an answer. In the absence of denial, the subsequent use of a related question treated the 'boredom' mentioned in the first part as a foundation for the pursuit of further information.

Notably, in both examples the recipient response slot was not taken up by the child and thus absence of a response was treated as tacit agreement. As aforementioned, however, in the wider data corpus agreement in some cases was verbally expressed.

Extract three: Family $1($ Prac $=$ Community psychiatric nurse $)$

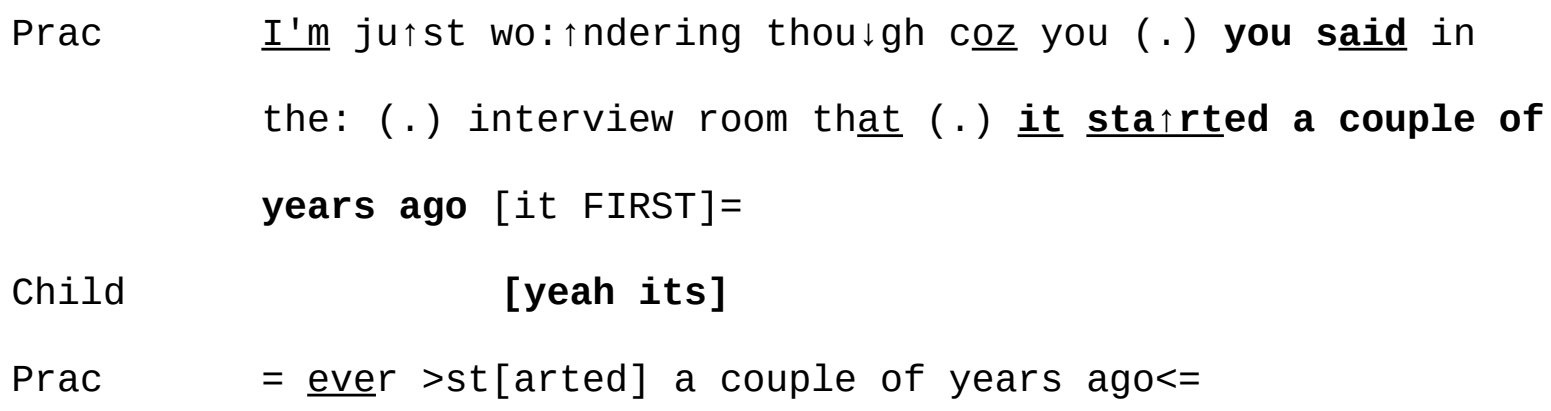


Prac: = so why $\uparrow$ do you think it it started the $\downarrow$ n?

Child January it could be sorting out changing thiings( $\odot .7 \odot)$

I th $\downarrow$ ink it could be like (.) say $\uparrow$ ing me (.) is coz like

(.) I dunno ( $\odot .63)$ chan $\uparrow$ ging schools an th $\downarrow$ at $l i \downarrow k e$

In this extract at the end of the first part of the sequence 'you said in the interview room that it started a couple of years ago' the child verbally responded by agreeing with the practitioner. This agreement occurred in overlap with the end of the practitioner's turn of talk. Arguably, the child appeared to have treated this moment as a transition relevance place. By referring to an earlier part of the assessment the practitioner made relevant the original version, which occurred 'in the interview room'. Although the child provided an acknowledgement by expressing agreement 'yeah', the practitioner repeated the phrase again. Once more the recipient response came in overlap to the practitioner's turn, orienting to the existence of a recipient response slot. Thus, the practitioner consolidated the now shared agreement regarding the suggested premise from the reflected speech 'it started a couple of years ago'. As with the previous two examples this provided a foundation for the subsequent question-answer adjacency pair.

\section{The functions of reflected speech}

Usually in the sequential organisation of talk recipients design what they say to respond to turn of talk that occurred immediately before it (Sacks, 1992). It is therefore difficult for a speaker to return to an earlier topic of conversation without doing some interactional work. Thus, we propose that the 'you said' sequence has a number of functions; 1 ) to indicate that the re-introduction of the topic was being made relevant again; 2) to soften the otherwise 
potentially challenging nature of the forthcoming question and; 3) to elicit clarification and/or elaboration (this is summarised in table 2).

Table 2: The three functions of the 'you said' sequence

1. To reintroduce a topic from earlier.

2. To soften a forthcoming question.

3. For clarification and elaboraton.

First, it is evident that one of the interesting functions of reflected speech was that the practitioner who introduced it selected from numerous topics covered in the assessment to that point. However, for client-focused practitioners in this institutional environment the benefit of using reflected speech was that it presented the aforementioned chosen topic as being initially the client's concern, rather than the practitioner's. Second, in the context of child mental health assessments the use of 'you said' functioned to soften the otherwise potentially challenging nature of the forthcoming question. This specific selection provided a platform for the practitioner to request further elaboration about what had already been introduced. In a similar way to how formulations are used by therapists to propose a summative version of what has the client has said (Ekberg and LeCouter, 2014), reflected speech also functions to propose a current basis of shared knowledge in order to proceed. However, in comparison to formulations which tend to be a précis of a number of preceding turns of talk, reflected speech had a highly selective function of identifying a single previous turn. Third, in these instances, another function of the sequence was to elicit clarification and/ or elaboration. In other words, the return to the initial topic had the purpose of providing a platform for eliciting further detail. 
Mum

${ }^{\circ} \mathrm{umhm}^{\circ}$

Prac

in in the house is it an older $\downarrow$ sister or younger

Child

Younger

In the recipient response slot in the extract above an acknowledgement was provided by the mother 'umhm', which indicated agreement that the child did have a sister. The third part of the sequence functioned to establish whether the child's sister was older or younger. This simplicity of the question was facilitated by offering an either-or choice (see Antaki \& O’Reilly, 2014). This type of questioning did not open up a topic or pursue detail, but simply requested a short response that directly attended to what was being asked.

Within the environment of initial assessments, the issue of elaboration appeared to be intrinsic to the information-seeking process. We have noted that the 'you said' sequence provided an important mechanism for practitioners to seek elaboration about particular therapy/diagnosis relevant topics and issues.

Extract five: Family $18($ Prac $=$ Psychiatrist $)$

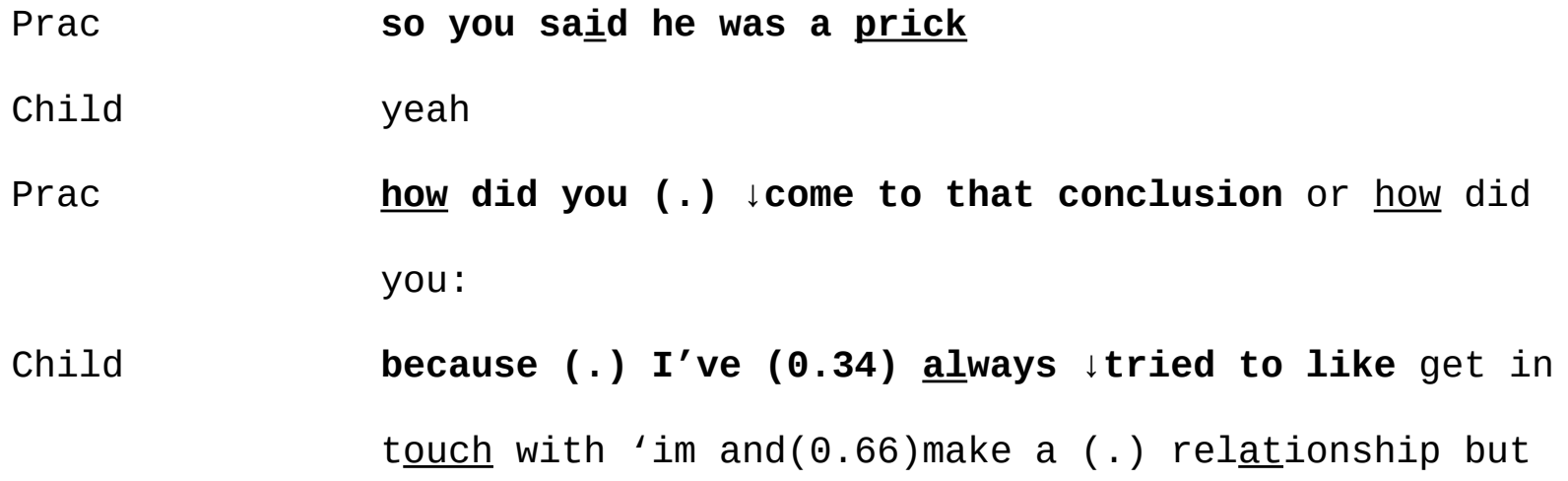




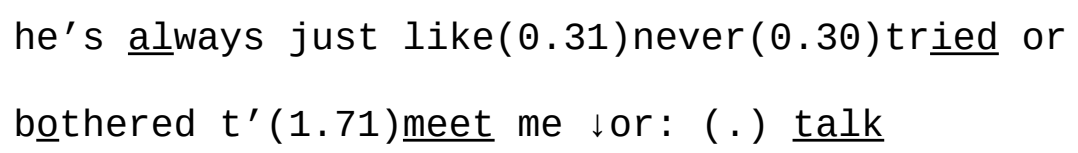

This extract provides another example of verbal agreement in the recipient-response slot. This provided a foundation for the third part of the sequence which was to initiate a questionanswer adjacency pair. This elaboration was solicited through a 'how' prefaced question 'how did you come to that conclusion'. Notably, the reference to 'that' in this question, is an example of what Sacks (1992) referred to as a 'tying rule' whereby 'that' is sequentially understood to be related to the prior turn of talk; which in this case was the reflected speech unit 'you said he was a prick'. Thus, by anchoring this question to the reflected speech, the practitioner was successful in eliciting further information from the child about that specific topic.

Extract six: Family $6($ Prac $=$ Psychiatrist $)$

Prac so when you $\downarrow$ said that you were going to take a $\downarrow$ knife to yourself

$(0.99)$

Prac yeah?

$(1.15)$

Prac what were you \hoping would happen?

Child erm (2.45) f::or me to $\downarrow$ actually kill my $\downarrow$ self

In all previous extracts the reflected speech unit was re-invoked from an earlier incantation within the same session. However, the reflected speech in the first line of this extract; 'you said that were going to take a knife to yourself', was not actually verbalised within this 
session. Thus, there is uncertainty about whether the phrase was actually used by the child in another environment. Nevertheless, the practitioner treated it as something that the child had said at some previous point. Notably, in this extract there are two recipient-response slots. Although the child's non-response in the first recipient-response slot (0.99 second pause) could have been treated as tacit agreement, the practitioner actively sought more explicit agreement from the child by using an additional tag question 'yeah?'. This precipitated the second recipient-response slot. Although again the child did not verbally respond, the practitioner moved onto the third part of the sequence by asking a follow-up question 'what were you hoping would happen'. Arguably the unusual feature of two recipient-response slots may relate to the less concrete certainty about what the child said as the initial uttering of the topic did not happen within the session. Given the potentially challenging nature of the reflected speech in this instance and the lack of original version within the current assessment, the agreement-seeking tag functioned to more definitely establish the first part as a basis for proceeding with the questions that followed. The success of this sequence as a mechanism for achieving elaboration relating to potentially difficult topics was demonstrated by the positive uptake where the child explained her rationale as 'for me to actually kill myself'.

\section{Comparative questions without 'you said' preface}

What has been demonstrated by the analysis is that the 'you said x' prefaced questions are effective in eliciting a relevant response, even where the topic of conversation is extremely sensitive. This preface allowed practitioners to revisit potentially difficult areas of conversation with the child. Although there were many questions in the data, and most of these were asked without a 'you said' preface, those that were prefaced in this way were 
always effective. The following examples are a small selection of questions asked without a 'you said' preface, which were not effective in eliciting information. We offer these to demonstrate the tricky business of asking children and young people about personal or emotionally sensitive areas of their lives and to demonstrate that non 'you said' prefaced questions may not always elicit relevant answers.

Extract seven: Family 19 (Prac - Assistant psychologist)

Prac W why do you think you've been referred today Joanne

Child dun [no: ]

Prac [(do you know) $]$

Child just coz: (1.60) dunno

Extract eight: Family 7 (Prac- Community Psychiatric Nurse)

Prac what about if $\downarrow$ people touch your $\uparrow$ stufff

Child $\quad$ I don't $\downarrow$ like that

Prac you don't $\downarrow$ like it $(0.55)$ how does it $\downarrow$ make you feel?

Child dunno(.) I $\uparrow$ don't like $\downarrow$ people going in my bag $\uparrow$ either

Extract nine: Family 6 (Prac - Psychiatrist)

Prac I wonder whether you $\downarrow$ think about $(0.23)$ what other kind of $\downarrow$ life $(0.75)$ might be $\uparrow$ like $(1.95)$ you know if you were with your $\downarrow$ sisters and brothers and $\uparrow$ stuff like $\downarrow$ that $(\odot .38)$ do you ever $\uparrow$ think about $\downarrow$ that?

Child: (7.78) ((child shrugs shoulders))

Prac: not $\downarrow$ sure $(0.30){ }^{\circ} \mathrm{O}^{\circ}($. ) $\underline{o} \downarrow k$

In the initial assessment the practitioners are virtual strangers to the child and yet part of the focus of the clinical task is to elicit personal, potentially difficult and sometimes emotionally sensitive information from the child. It can be quite tricky to elicit this type of information 
from children and what our earlier analysis demonstrated was that a 'you said’ preface worked well in acquiring such responses. These three examples illustrate some instances where practitioners asked questions that did not achieve a relevant response; in extract seven the child used the response 'I dunno'; in extract eight the child gave a response but not one which addressed the question; and in extract nine the child gave a non-verbal shrug of the shoulders following a substantial pause. These three examples demonstrate that without the 'you said' preface there is a risk that relevant responses may not be forthcoming.

\section{Discussion and conclusions}

The overarching thesis of this paper has been to illustrate how communications processes are crucial within therapeutic interactions, especially with children. The skill of demonstrating active listening is one that facilitates child-centred work and maintains engagement of children in therapy and assessment sessions. In particular, we have focused on how assessment progress may be achieved through specific kinds of question design that incorporate some of the principles of active listening. Certain question constructs have potential to facilitate a shared knowledge base, which is argued to be a helpful prerequisite to the pursuit of further information relevant to the assessment objectives. The use of reflected speech using the preface 'you said' has been the focus of this inquiry. Through conversation analysis, we have revealed a three-part interactional sequence.

The first part consisted of the preface 'you said' and the reflected speech, which functioned to propose a basis for shared knowledge. The second part consisted of the provision for a recipient-response slot, which sometimes resulted in active uptake from the child and on some occasions did not. However, proceeding onto the third part of the sequence was 
contingent upon either tacit or actual agreement being established. In other words, both silences and confirmation at the transition relevant place after completion of the first component were treated as agreements. The third part of the sequence consisted of the question-answer adjacency pair. The question was made possible because of the foundation built through the first and second parts.

In an environment where information gathering is the primary objective, the importance of effective structuring of question sequences is particularly pertinent. In the data presented the use of this sequence was shown to be effective in yielding assessment relevant information from children. Effectiveness as we have used the term referred to the children's responses to the practitioner's question being appropriate and relevant for the question, presenting a sufficient level of detail. Thus, the three-part sequence presented in this paper has been shown to function as a mechanism to legitimately reintroduce topics previously mentioned earlier in the conversation in order to seek clarification or elaboration. We noted that when this technique was used it was often an effective way of reintroducing a pertinent area for exploration, such as identifying when a particular behaviour began (extract 3) or understanding the motivations for the child's reactions (extract 1). One of the benefits of using this particular technique is that it retains a greater client-focus than if the practitioner had introduced the topic unilaterally (for example, extracts 7, 8 \& 9).

Previous research has shown that overcoming resistance and engaging children meaningfully can be challenging (Hutchby, 2002; O’Reilly \& Parker 2013). Additionally, research has shown that discussions about sensitive topics can be potentially troublesome and delicate (for example, Antaki, 2007). The poor management of this can result in therapeutic disalignment and in some cases therapeutic rupture. Careful and reflexive awareness of the potential for 
therapeutic rupture or disalignment is therefore an important skill to develop (Parker \& O’Reilly, 2013). The 'you said' sequence that we have identified offers a useful strategy for practitioners to facilitate alignment as it displays attentiveness to the client's narrative. This sequence also indicates the client's contribution is important enough to be revisited which can be validating for the child. Because the reintroduced topic is centred in the client's own words, this provides a platform for the practitioner to pursue further clarification or elaboration without seeming presumptuous. We argue that the reintroduction of reflected speech facilitates a catalytic process which brings the child's experiential knowledge into an equal domain with the practitioner's expert knowledge. Indeed, the use of the client's own words in effect legitimises the topic as a permissible line of inquiry and recognises the value of the child's perspective.

We recognise that the data set presented was drawn from a particular institutional context of child mental health assessments and as such the findings are likely to have a stronger practical application to the field of mental health than other institutional setting. However, the recommendations and messages provided may translate to other contexts where children are asked questions about their lives. Furthermore, with the collection of naturally occurring data, researchers are unable to influence the content of the data collected, such as requiring practitioners to ask about specific topics from the research agenda. Nonetheless, it is wellestablished that research should be directly relevant to service implementation and development (World Health Organization, 2013). Naturally occurring data is an excellent resource for investigating practitioner interventions in real world settings and as such its findings have direct relevance to practice. 
There are a number of practical implications relating to the appreciation of the impact of question design for clinical practice. It is essential that practitioners are reflective about the design and impact of the kind of question sequences that they employ. We suggest that our findings will be valuable within the mental health domain in order to help practitioners to design questions in ways that will lead to optimal efficacy in gathering information. In particular, reintroducing reflected speech by using the simple conversational preface of 'you said' is argued to be a highly effective strategy for clinical practice. For practitioners working within services that dictate resource constraints of time, personnel and finances, it is pragmatic to consider ways in which practitioners can become more skilful in their role as information gatherers during assessment appointments. To this end, we recommend that practitioners engage with the evidence on communicating with children, and in particular seek out additional CA research that focuses on this area. However, these findings also have relevance for professionals working in a range of different settings, such as education, social care and the criminal justice system. Reflected speech is a ubiquitous aspect of talk in many different kinds of institutional settings and thus these findings demonstrate its value as a question-preface for eliciting information.

Additionally, we promote the use of findings from CA research in training programmes designed for mental health practitioners as an empirical platform for practical skills development in communication. For example, all mental health professionals undertake initial qualifications in their respective disciplines, such as medical or nursing education, doctorates in clinical psychology, qualifications in counselling; and communication skills typically feature on the curricula to different extents. With the greater emphasis on engaging with evidence to inform practice, trainers could take the opportunity to utilise CA findings to 
facilitate teaching and learning in relation to communication skills. This could be further expanded in terms of continuing professional development.

In conclusion, we propose that by using the client's own words and offering reflected speech, sessions can be designed to be more client-centred. This has greater potential to promote engagement, which is particularly important in settings where effective communication with children is paramount. The use of the 'you said' sequence has been demonstrated to be an effective technique for eliciting information from children. 


\section{References}

Antaki, C. (2007). Mental-health practitioners' use of idiomatic expressions in summarising client's accounts. Journal of Pragmatics, 39, 527-541.

Antaki, C., \& O’Reilly, M. (2014). Either/or questions in psychiatric assessments: the effect of the seriousness and order of the alternatives. Discourse Studies, 16(3) 327345

Bone, C., O’Reilly, M., Karim, K., and Vostanis, P. (2014). “They’re not witches...”: Young children and their parents’ perceptions and experiences of Child and Adolescent Mental Health Services. Child: Care, Health and Development, 41(3), 450-458.

Chu, B. \& Kendall, P. (2004). Positive associations of child involvement and treatment outcome within a manual-based cognitive behavioral treatment with anxiety. Journal of Consulting and Clinical Psychology, 72, 821-829

Drew, P., Chatwin, J., \& Collins, S. (2001). Conversation analysis: A method for research into interactions between patients and health-care professionals. Health Expectations, $4(1), 58-70$

Ekberg, K., \& LeCouteur, A. (2014). Co-implicating and re-shaping clients’ suggestions for behavioural change in cognitive behavioural therapy practice. Qualitative Research in Psychology, 11, 60-77.

Hartzell, M., Seikkula, J., \& von Knorring, A. (2010). Parent’s perception of their first encounter with child adolescent psychiatry. Contemporary Family Therapy, 32, 273289.

Heritage, J. (1984). A change-of-state token and aspects of its sequential placement. In J. M. Atkinson, \& J. Heritage (Eds.), Structures of Social Action: Studies in Conversation Analysis (pp. 299 - 345). Cambridge: Cambridge University Press. 
Holt, E. (1996). Reporting on talk: the use of direct reported speech in conversation. Research on Language and Social Interaction, 29, 219-245

Hutchby, I., (2005). ‘Active listening': formulation and the elicitation of feelings-talk in child counselling. Research on Language and Social Interaction, 38(3), 303-32

Hutchby, I. (2002). Resisting the incitement to talk in child counselling: aspects of the utterance 'I don’t know'. Discourse Studies, 4(2): 147-168

Hutchby, I., \& Wooffitt, R. (2008). Conversation Analysis (Second Edition). Oxford: Blackwell Publishers.

Jefferson, G. (2004). Glossary of transcript symbols with an introduction. In G. H. Lerner (Ed.), Conversation Analysis: Studies from the First Generation (pp. 13-31). Amsterdam: John Benjamins.

Kazdin, A. (2004). Psychotherapy for children and adolescents. In M. Lambert (Ed.), Bergin and Garfield’s Handbook of Psychotherapy and Behaviour Change (pp. 543-589). New York: John Wiley

Mash, E., \& Hunsley, (2005). Special section: Developing guidelines for the evidencebased assessment of child and adolescent disorders. Journal of Child and Adolescent Psychology, 34 (3), 362-379.

McCabe, R., Heath, C., Burns, T., \& Priebe, S. (2002). Engagement of patients with psychosis in the consultation: conversation analytic study. British Medical Journal, 325:1148-51

O’Reilly, M., Karim, K., \& Kiyimba, N. (2015). Question use in child mental health assessments and the challenges of listening to families. British Journal of Psychiatry Open, 1 (2), 116-120

O’Reilly, M., Karim, K., Stafford, V., \& Hutchby, I. (2015). Identifying the interactional 
processes in the first assessments in child mental health. Child and Adolescent Mental Health, 20(4), 195-201.

O’Reilly, M., \& Lester, J. (2015: Eds). The Palgrave handbook of child mental health. Basingstoke, Palgrave MacMillan.

O’Reilly, M. \& Parker, N. (2013). 'You can take a horse to water but you can’t make it drink’: Exploring children’s engagement and resistance in family therapy. Contemporary Family Therapy, 35(3), 491-507.

Parkin, A., Frake, C., \& Davison, I. (2003). A triage clinic in a Child and Adolescent Mental Health Service. Child and Adolescent Mental Health, 8(4), 177- 183

Parker, N., \& O’Reilly, M. (2013). Reflections from behind the screen: avoiding therapeutic rupture when utilising reflecting teams. The Family Journal: Counseling for Couples and Families, 21(2), 170-179.

Parker, N. \& O’Reilly, M. (2012). 'Gossiping’ as a social action in family therapy: The pseudo-absence and pseudo-presence of children. Discourse Studies, 14(4) 1-19.

Potter, J. (2002). Two kinds of natural. Discourse Studies. 4 (4), 539-542.

Sacks, H. (1992). Lectures on Conversation (Vols. I \& II, edited by G. Jefferson). Oxford: Basil Blackwell

Sacks, H., Schegloff, E., \& Jefferson, G. (1974). A simplest systematics for the organization of turn-taking for conversation. Language, 50, 696-735

Stivers, T. (2002). Presenting the problem in pediatric encounters: "symptoms only" versus “candidate diagnosis” presentations. Health Communication, 14 (3), 299-338.

Stivers, T. (2001). Negotiating who presents the problem: Next speaker selection in pediatric encounters. Journal of Communication, June: 252- 282.

World Health Organization (2013). Mental health action plan 2013-2020. Geneva: WHO 
Wooffitt, R., \& Allistone, S. (2008). Participation, procedure and accountability: 'You said' speech markers in negotiating reports of ambiguous phenomena. Discourse Studies, 10(3), 407-427

Ziolkowska, J. (2009). Positions in doctors’ questions during psychiatric interviews.

Qualitative Health Research, 19(11), 1621-1631 